\title{
New method: the intravital videomicroscopic characteristics of the microcirculation of the urinary bladder in rats
}

Received: 14 November 2001 / Accepted: 21 March 2002 / Published online: 17 May 2002

(C) Springer-Verlag 2002

\begin{abstract}
Intravital fluorescence microscopy (IVM) is a widely used method to study the microcirculation in several organs. Our aim was to develop a standard rat model to evaluate the microcirculatory characteristics of the urinary bladder under physiological pressure conditions using the most advanced fluorescence videomicroscopic techniques. Spraque-Dawley rats were used after filling their bladders with a constant volume of saline solution. The intravesical pressure was continuously monitored. The bladder was positioned on a specially designed stage and IVM measurements were made at the beginning, the 90th, 120th and 180th min. Arteriolar and venular diameters, functional capillary density, venular red blood cell velocity, arteriolar and venular macromolecular leakage and leukocyte-endothelial cell interactions (observation of rolling and firmly adherent leukocytes) were quantitatively assessed by a computer assisted analysis system. Neither microcirculatory parameters nor the intravesical pressure changed significantly during the observation period of $180 \mathrm{~min}$ using constant filling volume. We successfully established a new, well functioning and reproducible model to study the microcirculation of the rat bladder using intravital fluorescent microscopy.
\end{abstract}

Keywords Urinary bladder - Microcirculation · Intravital fluorescence microscopy $\cdot$ Rats

\section{Introduction}

The microcirculation consists of blood vessels with an outside diameter of less than $300 \mu \mathrm{m}$, the blood and its

\footnotetext{
Z. Bajory $(\bowtie) \cdot$ J. Hutter $\cdot$ F. Krombach $\cdot$ K. Messmer

Institute for Surgical Research, University of Munich,

Klinikum Grosshadern, Munich, Germany

E-mail: bajory@freemail.hu

Tel.: + 36-62-490590

Fax: + 36-62-420886

Z. Bajory

Department of Urology and Institute of Surgical Research, Albert Szent-Györgyi Medical Center,

University of Szeged, Kalvaria sgt. 57, 6725 Szeged, Hungary
}

components in these vessels, and the surrounding interstitial tissue. The primary function of the microcirculation proper is the convective transport of substrates to and metabolic products from the cells in a tissue. The local adaptation of these transport conduits to the ever changing metabolic and functional needs of the tissue is thus a prerequisite for a physiologically relevant microcirculation.

Until recently, technical limitations made it difficult to observe and measure microcirculatory variables such as small blood vessel diameter, red blood cell velocity, microvascular permeability and the state of leukocyte activation. Over the last decade, and in particular over the last 5-7 years, considerable data have been accumulated in this area, primarily from various fields of animal research, using, for instance, fluorescence videomicroscopy $[11,12]$. The microcirculation of many organs has been observed in vivo under physiological and pathophysiological conditions $[5,6,7,8,9,10,11,12]$, new perspectives for tumor and tissue transplantation research have been opened using fluorescence microscopy [12] and microcirculatory observations of conscious animals has become possible [3, 12]. Most of the characteristic details of the microcirculatory response to various pathophysiological stimuli of the urogenital tract, however, remain to be elucidated. In particular, we aimed to develop a reproducible model to study urinary bladder microcirculation, for which only very few microcirculatory data are at present available. To study the microcirculatory changes of different organs in vivo is a very important and interesting field of experimental research on inflammatory reactions, and intravital videomicroscopy is employed as the gold standard to monitor it.

\section{Materials and methods}

Animals

The experiments were performed after government approval under the guidelines of the National Institutes of Health (NIH publication No. 86-23, revised 1984). 
Eight male Spraque-Dawley rats from our laboratory $(N=8$, average weight $250 \mathrm{~g}$.) were housed in an environmentally controlled room with a 12-h light-dark cycle, with free access to water and standard laboratory pellet food $(18,000 \mathrm{IU} / \mathrm{kg}$ vitamin A, $1,280 \mathrm{IU} /$ vitamin $\mathrm{D}_{3}, 120 \mathrm{mg} / \mathrm{kg}$ vitamin E; Ssniff Spezialdiäten, Soest, Germany).

\section{Surgical procedure and experimental protocol}

After sodium pentobarbital (Narcoren; Merial, Hallbergmoos, Germany) anesthesia ( $45 \mathrm{mg} / \mathrm{kg}$, intraperitoneally) and premedication with atropine sulfate $(0.1 \mathrm{mg} / \mathrm{kg}$, subcutaneously; Braun, Melsungen, Germany) the animals were placed in a supine position on a heating pad for maintenance of the normal body temperature during the experiment [8]. All of the surgical procedures were made under a surgical microscope (Leica M651; Bensheim, Germany). Polyethylene catheters (ID $0.28 \mathrm{~mm}$., OD $0.61 \mathrm{~mm}$.; SIMS Portex, Hythe, UK) were inserted into the left carotid artery and jugular vein to measure the mean arterial pressure (MAP), heart rate (Plugsys; Hugo Sachs Elektronik, March, Germany) and for the injection of fluorescence dyes for intravital videomicroscopy (IVM). The animals were intubated (Abbocath-T; 13G; ABBOTT, Sligo, Ireland) through a tracheotomy.

After a midline laparotomy the bladder was exposed and the median umbilical ligament was cut near the bladder. The urethra was ligated with 4-0 Perma-Hand Silk (Johnson and Johnson, Brussels, Belgium). The two ureters were cut in the middle to prevent the bladder from overfilling during the experiment. A polyethylene catheter (ID $0.28 \mathrm{~mm}$, OD $0.61 \mathrm{~mm}$; SIMS Portex) was inserted into the bladder at the dome in order to fill the bladder and to measure the intravesical pressure. The bladder was first emptied and then was filled with $0.5 \mathrm{ml}$ of bodywarm $0.9 \% \mathrm{NaCl}$ solution. The intravesical pressure was continuously measured with a pressure monitor (Plugsys).

The bladder was exteriorized drawing gently by the stump of the umbilical ligament and was placed onto a specially designed stage for IVM (Fig. 1). After the IVM measurements, the bladder was replaced into the abdomen and the abdominal wall was closed by clamps until the next IVM measurement. IVM were made at the beginning and the 90 th, 120th and 180th min after the preparation.

Afterwards the animals were killed with an overdose of sodium pentobarbital.

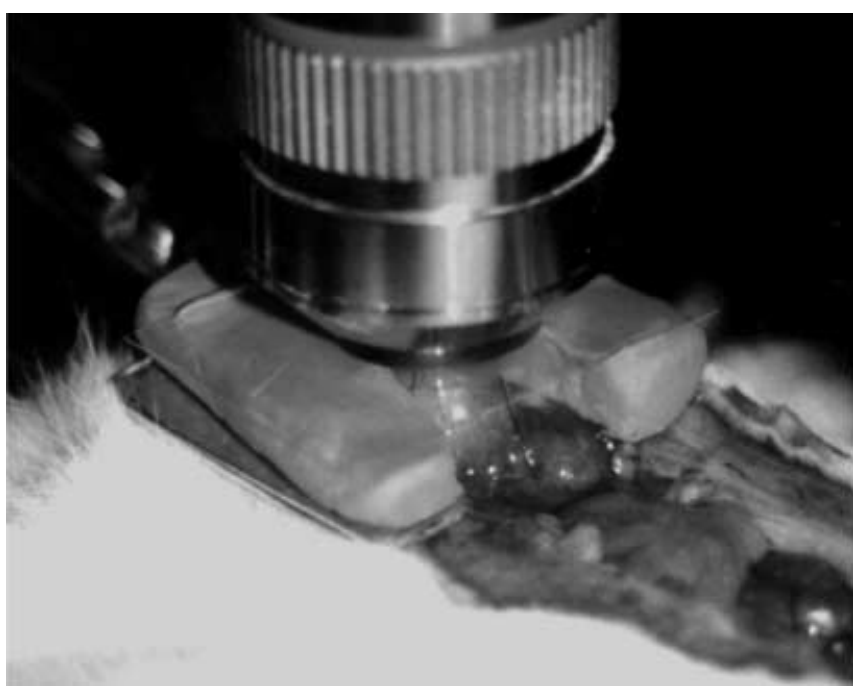

Fig. 1. The urinary bladder on a special stage under the objective of the fluorescence microscope
Intravital videomicroscopy

Contrast enhancement was achieved by intravenous injection of fluorescein isothiocyanate (FITC)-labeled albumin (mol. wt. 70,000, $0.2 \mathrm{ml}$.; Sigma, St. Louis, Mo., USA) for the plasma and rhodamine $6 \mathrm{G}$ (mol. wt. $479,0.2 \%, 0.1 \mathrm{ml}$; Sigma) for the leukocyte labeling $10 \mathrm{~min}$ before the IVM evaluations. The microcirculatory network of the examined organ was visualized by using a high-resolution, modified Zeiss-Orthoplan fluorescence intravital microscope attached to a Ploemo-Pak illuminator with an I2/3 blue [exitation filter (Ex): $495 \mathrm{~nm}$, emission filter (Em): $515 \mathrm{~nm}$ ] and N2 green (Ex: $525 \mathrm{~nm}$, Em: $555 \mathrm{~nm}$ ) filter block (Leitz, Wetzlar, Germany). The microcirculation was analyzed using an epi-illumination technique. With a $25 \times$ water immersion objective (W $25 \times / 0.6$; Leitz) the magnification was $540 \times$ on the video screen (Sony, Tokyo, Japan). The microscopic images were recorded by a charge-coupled device video camera (FK 6990; Pieper, Schwerte, Germany) attached to an S-VHS video recorder (BR-S920E; JVC, Tokyo, Japan) for later off-line computer assisted evaluation $[2,16]$.

\section{Video analysis}

The quantitative assessment of microcirculatory parameters was performed off-line by analysis of the videotaped images using a computer-assisted analysis system (CAMAS, Dr. H. Zeintl, Heidelberg, Germany) [2.]. Arteriolar and venular diameter $(\mu \mathrm{m})$ (Fig. 2), functional capillary density (FCD, length of erythrocyte perfused nutritive capillaries per observation area of $1 / \mathrm{cm}$ ) (Fig. 3), venular red blood cell velocity (RBCV, $\mathrm{mm} / \mathrm{s})$ and macromolecular leakage (ratio of extravascular fluorescence intensity and intravascular fluorescence intensity) were determined in five observation fields of the muscular layer [16]. Leukocyteendothelial cell interactions[1] were analyzed within five venules $(n=5)$ per animal with respect to rolling and adherent leukocytes (Fig. 4). Rolling leukocytes were defined as cells moving significantly more slowly than the red blood cells moving in the centerline of the vessels and are given as the number of rolling leukocytes per vessel diameter (mm) per second. Adherent leukocytes (stickers) were identified in each vessel segment as cells that did not detach from the endothelial lining within $30 \mathrm{~s}$ and are given as number of cells per square millimeter of endothelial surface, calculated from the diameter and length of the venule segment, assuming cylindrical geometry [2, 17].

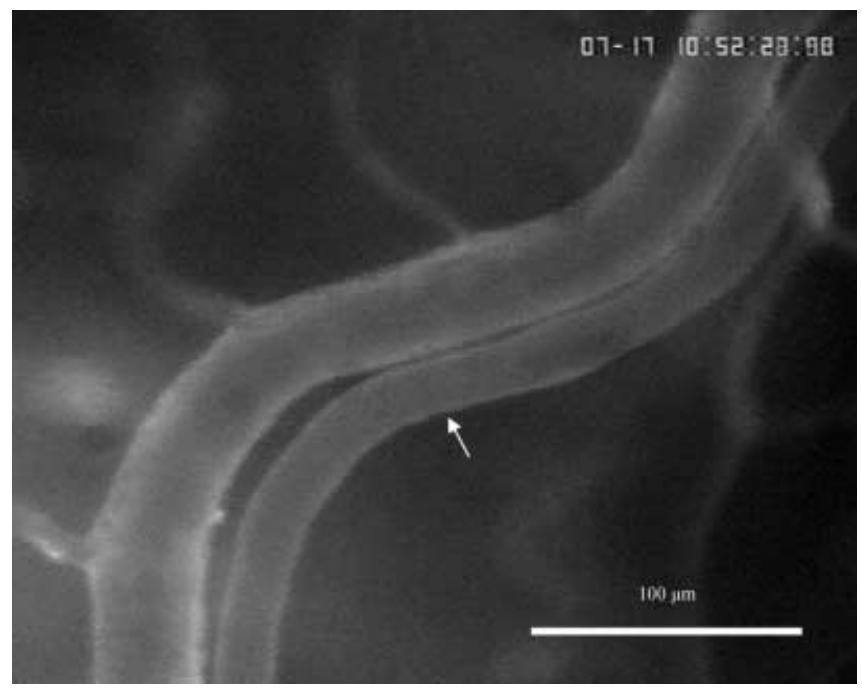

Fig. 2. An arteriole (arrow) and a venule of the muscular layer of the bladder. FITC-albumin plasma labeling 


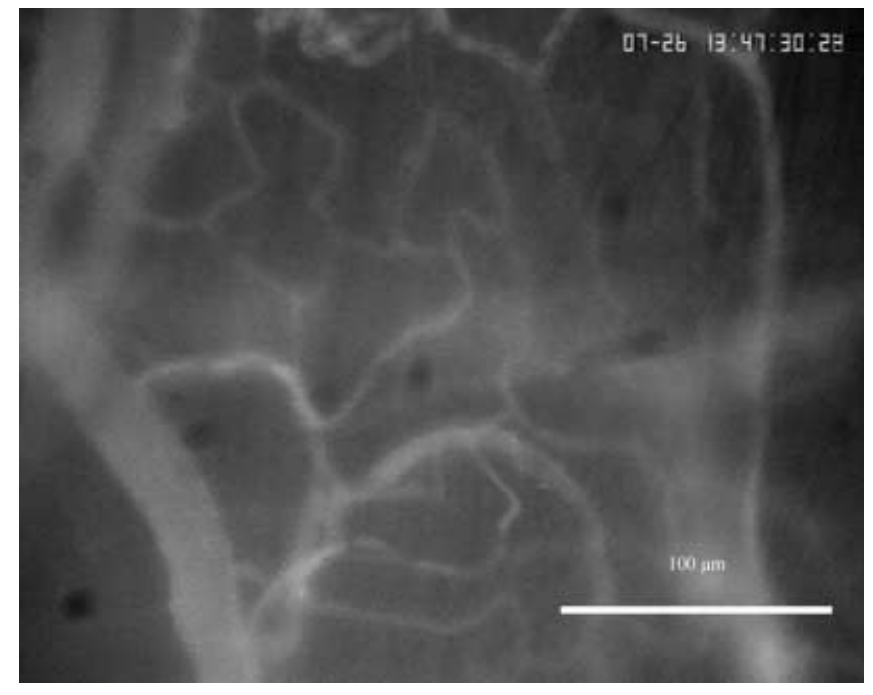

Fig. 3. The capillary network in the muscular layer of the bladder wall. FITC-albumin plasma labeling

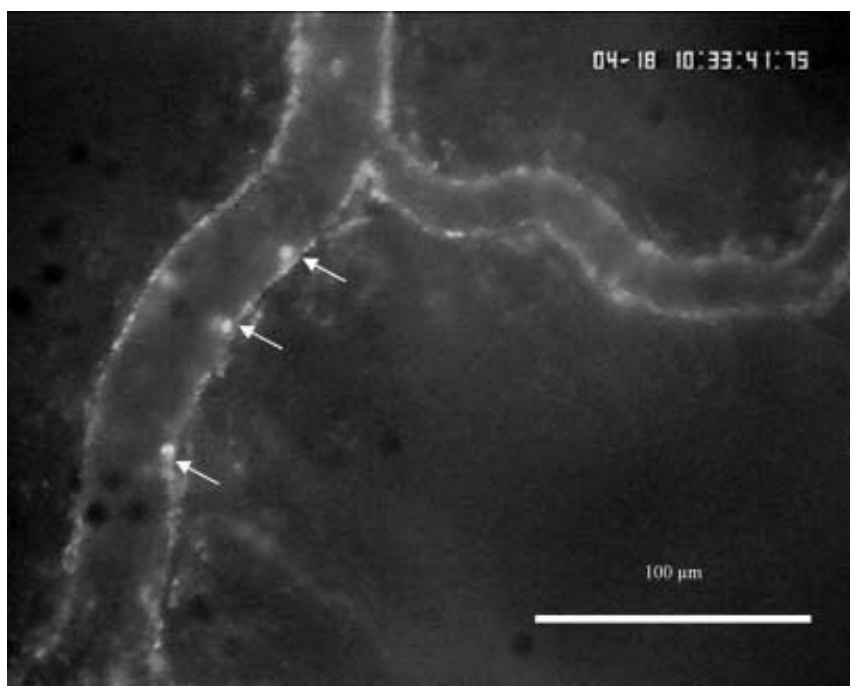

Fig. 4. Venules of the muscular layer of the urinary bladder. Rhodamine-6G labeled leukocytes (arrows) rolling on the wall of the venule

\section{Statistics}

The data derived from parameters display a non-gaussian distribution. The analysis was performed with a statistical software package (SigmaStat 2.0 for Windows, Jandel Scientific, Germany). Friedman repeated measures for analysis of variance was applied for multiple comparisons between the different time points. Means $\pm \mathrm{SD}$ are given. $P<0.05$ was considered significant.

\section{Results}

After filling the bladder with $0.5 \mathrm{ml}$ saline solution, the intravesical pressure was $9 \pm 2 \mathrm{mmHg}$ and did not change significantly during the observation period. The macrohemodynamic parameters were within physiolog- ical limits during the experiments and did not change significantly (data not shown).

There were no significant changes in any microcirculatory parameter during the whole $180 \mathrm{~min}$ period compared to the control values (Table 1).

\section{Discussion}

In spite of the fact that the use of the intravital videomicroscope has become increasingly popular for studying organ microcirculation $[3,5,6,7,8,9,10,11$, 12] very few reports in the literature deal with the intravital videomicroscopic evaluation of urinary bladder microcirculation. Our aim was to develop a reproducible rat model to precisely characterize the microvascular perfusion parameters of the urinary bladder in a standardized basic state, using a high quality fluorescence videomicroscope and the well established epi-illumination technique.

The rat is one of the most commonly used animals in the field of the microcirculatory research. The urinary bladder of the rat is very similar to that of humans, both anatomically and functionally [4, 13]. The main branches of the cystic arteries and large veins form an adventitial-serosal plexus which supplies the capillary network of the muscular layer and sends long perpendicular vessels to the mucosal plexus. Apart from a few vascular connections between these two plexus, the submucosa is generally avascular. The muscular layer contains tortuous arterial and venous branches mostly derived from the adventitial-serosal plexus. In comparison with the extreme density of the subepithelial capillary network, the capillaries of the muscularis are poorly developed [13]. The microcirculatory network of the rat bladder can be visualized within the maximal depth of $300 \mu \mathrm{m}$ of the muscular layer with IVM and can be distinguished from the vessels of other layers by their specific architecture.

The urinary bladder of the rat was chosen for our study because its thin tissue allows for high resolution images and precise quantification of the microcirculatory parameters. To study the microcirculation of the bladder with IVM in an animal smaller than the rat would be disproportionately more difficult and the scatter resulting from technical problems would require the use of much larger experimental groups.

Reed et al. described some microcirculatory changes of the urinary bladder after photodynamic therapy using a fluorescence microscopic technique. The microcirculation was observed by transmitted light. In their study the organ was placed into a plexiglass tissue bath, fixed by a suture on the bladder surface. The bladder was almost empty during the examination and the intravesical pressure and volume were not determined. Only the diameters of the red blood cell column and the presence or absence of platelet thrombi were observed as microcirculatory parameters [14]. 
Table 1. Microcirculatory characteristics of the urinary bladder in rats. Data are shown as means $\pm \mathrm{SD}$ of eight animals $(N=8, n=5, m=5: N$ is the number of animals, $n$ the number of observed vessels per animal and $m$ the number of fields of interest per animal). There was no statistically significant difference between the measurements using $P=0.05$ as the level of significance

\begin{tabular}{lcccc}
\hline & Base line & $90 \mathrm{~min}$ & $120 \mathrm{~min}$ & $180 \mathrm{~min}$ \\
\hline Rolling leukocytes $/ \mathrm{mm} / \mathrm{s} n=5$ & $6.0 \pm 1.8$ & $7.5 \pm 1.3$ & $8.8 \pm 2.8$ & $8.5 \pm 3.9$ \\
Adherent leukocytes $/ \mathrm{mm}^{2} n=5$ & $12.7 \pm 17.3$ & $20.0 \pm 9.5$ & $28.8 \pm 17.8$ & $26.1 \pm 13.8$ \\
Venular macromolecular & $0.74 \pm 0.10$ & $0.76 \pm 0.12$ & $0.78 \pm 0.11$ & $0.79 \pm 0.10$ \\
$\quad$ leakage $n=5$ & & & & \\
Arteriolar macromolecular & $0.77 \pm 0.08$ & $0.79 \pm 0.08$ & $0.81 \pm 0.08$ & $0.80 \pm 0.06$ \\
$\quad$ leakage $n=5$ & $1.2 \pm 0.1$ & $1.3 \pm 0.1$ & $1.2 \pm 0.1$ & $1.1 \pm 0.1$ \\
RBCV $n=5(\mathrm{~mm} / \mathrm{s})$ & $141.6 \pm 12.2$ & $138.3 \pm 9.8$ & $132.2 \pm 6.0$ & $136.2 \pm 9.2$ \\
FCD $m=5(/ \mathrm{cm})$ & $41.2 \pm 22.1$ & $39.2 \pm 14.2$ & $37.2 \pm 15.3$ & $40.2 \pm 3.4$ \\
Venular diameter $n=5(\mu \mathrm{m})$ & $40.7 \pm 11.1$ & $37.7 \pm 8.8$ & $44.5 \pm 18.2$ & $39.5 \pm 11.0$ \\
Arteriolar diameter $n=5(\mu \mathrm{m})$ & & &
\end{tabular}

In an other study, Young et al. used an in vivo preparation to evaluate the effects of histamine, norepinephrine and prostaglandins on the arterioles and venules of the rat urinary bladder. In their model the bladder was illuminated by light transmitted along a borosilicate glass without the use of fluorescein dye [15]. Consequently, data comprising all of the relevant microcirculatory parameters of the urinary bladder under physiological conditions have not been available to date.

A large amount of data exists on the microcirculation of other organs in various species $[3,5,6,7,8,9,10,11$, 12]. Among these, the microcirculatory parameters of the intestine and the mesentery are most suitable for a comparison to our results.

Massberg et al. described the microcirculatory characteristics of the small intestine in the mouse. In their preparation, the FCD of the muscular layer was $226 \pm 21 / \mathrm{cm}$ under normal conditions. The leukocyteendothelial cell interaction was also analyzed. Under physiological conditions only few white blood cells were found rolling along or firmly adherent to the endothelium $\left(1 \pm 0.2\right.$ cells $/ \mathrm{mm} / \mathrm{s}$ and $18 \pm 7$ cells $/ \mathrm{mm}^{2}$, respectively) [9].

Boros et al. investigated the microcirculation of the small intestine in rats. The RBCV was $0.88 \pm 0.17 \mathrm{~mm} / \mathrm{s}$ and the FCD was $213 \pm 25 / \mathrm{cm}$, values similar to the respective values in the mouse. In the longitudinal muscle layer, the respective values were $164 \pm 15 / \mathrm{cm}(\mathrm{FCD})$ and $0.78 \pm 0.09 \mathrm{~mm} / \mathrm{s}$ (RBCV). The number of firmly adherent leukocytes (cells $/ \mathrm{mm}^{2}$ ) in the collecting venules was 8-10 under normal conditions [2].

The microcirculation of the rat mesentery also was studied by IVM. In contrast to the intestine, which is very similar to the bladder, the microcirculatory characteristic of the mesentery differs from the bladder in several aspects. Under control conditions, Kurose et al. found that the RBCV is $3.12 \pm 0.18 \mathrm{~mm} / \mathrm{s}$ and the leukocyte adherence is about $5 \pm 2$ cells $/ 100 \mu \mathrm{m}$ vessel length. The higher flow can be explained by the larger vessel diameters, however the values of leukocyte adherence were similar to those found in the bladder [7].

In our present study, we aimed to develop a standard rat model for further microcirculatory investigations and to describe the physiological characteristics of the microcirculation of the urinary bladder. We filled the urinary bladder with a constant volume of saline and measured the intravesical pressure during the whole $180 \mathrm{~min}$ examination period. The bladder was placed - only for the time of the microscopic observation - onto a specially designed stage for the best visualization of the microcirculatory network and between the measurements it was returned to its natural position. All relevant microcirculatory parameters were determined and showed no significant changes during the observation period. There is a slight tendency for leukocyte-endothelial cell interaction and of FCD to deviate from baseline values at the subsequent measurements. The anesthetic regimen chosen for this study could be one explanation. Atropine is widely used in small animals for premedication. So far, there are no controlled studies on the influence of this drug on the microcirculation, but given that baseline measurements were obtained about $1 \mathrm{~h}$ after atropine administration and the half live of atropine is $3-4 \mathrm{~h}$ when given i.m., the slight differences can not be explained by the use of this drug. Likewise, the i.p. administration of pentobarbital can be assumed to effect the macrocirculation. However, the macrohemodynamic parameters remained unchanged throughout the experiments.

In conclusion, we successfully established a novel, reliable rat model to study the microcirculation of the urinary bladder using a modern intravital fluorescence videomicroscope and the epi-illumination fluorescence technique, as a very sensitive and applicable method.

This newly developed, standardized microcirculatory model can serve as the basis for further investigations using the IVM for better understanding the basic pathophysiology of this organ at the microcirculatory level.

Acknowledgements This study was supported by the European Urological Scholarship Programme (EUSP) of the European Association of Urology (EAU) and OTKA F 032069

\section{References}

1. Adams DH, Shaw S (1994) Leucocyte-endothelial interactions and regulation of leucocyte migration. Lancet 343: 831

2. Boros M, Massberg S, Baranyi L, Okada H, Messmer K (1998) Endothelin 1 induces leukocyte adhesion in submucosal venules of the rat small intestine. Gastroenterology 114: 103

3. Endrich B, Asaishi K, Goetz A, Messmer K (1980) Technical report - a new chamber technique for microvascular studies in unanesthetized hamsters. Res Exp Med (Berlin) 177: 125 
4. Inoue T, Gabella G (1991) A vascular network closely linked to the epithelium of the urinary bladder of the rat. Cell Tissue Res 263: 137

5. Kamler M, Lehr HA, Barker JH, Saetzler RK, Galla TJ, Messmer K (1993) Impact of ischemia on tissue oxygenation and wound healing: intravital microscopic studies on the hairless mouse ear model. Eur Surg Res 25: 30

6. Klar E, Endrich B, Messmer K (1990) Microcirculation of the pancreas. A quantitative study of physiology and changes in pancreatitis. Int J Microcirc Clin Exp 9: 85-101

7. Kurose I, Wolf R, Grisham MB, Granger N (1994) Modulation of ischemia/reperfusion-induced microvascular dysfunction by nitric oxide. Circ Res 74: 376

8. Massberg S, Boros M, Leiderer R, Baranyi L, Okada H, Messmer K (1998) Endothelin (ET)-1 induced mucosal damage in the rat small intestine: role of $\mathrm{ET}_{\mathrm{A}}$ receptors. Shock 9: 177

9. Massberg S, Eisenmenger S, Enders G, Krombach F, Messmer K (1998) Quantitative analysis of small intestinal microcirculation in the mouse. Res Exp Med 198: 23

10. Menger MD, Marzi I, Messmer K (1991) In vivo fluorescence microscopy for quantitative analysis of the hepatic microcirculation in hamsters and rats. Eur Surg Res 23: 158
11. Messmer K, Krombach F (1998) Microcirculation research in experimental surgery. Chirurg 69:333

12. Messmer K, Funk W, Endrich B, Intaglietta M (1984) The perspectives of new methods in microcirculation research. Prog Appl Microcirc 6: 77

13. Miodonski AJ, Litwin JA (1999) Microvascular architecture of the human urinary bladder wall: a corrosion casting study. Anat Rec 254: 375

14. Reed MWR, Schuschke DA, Ackerman DM, Harty JI, Wieman TJ, Miller FN (1989) The response of the rat urinary bladder microcirculation to photodynamic therapy. J Urol 142: 865

15. Young WFJr, Dey RD, Echt R (1979) Comparisons of prostaglandin vasoactive effects and interactions in the in vivo microcirculation of the rat urinary bladder. Microvasc Res 17: 1

16. Zeintl H, Tompkins WR, Messmer K, Intaglietta M (1986) Static and dynamic microcirculatory video images analysis applied to clinical investigations. Prog Appl Microcirc 11: 1

17. Zeintl H, Sack FU, Intaglietta M, Messmer K (1989) Computer assisted leukocyte adhesion measurement in intravital microscopy. Int J Microcirc Clin Exp 8: 293 